\title{
Recent Progress on Monotone Integrated Large Eddy Simulation of Free Jets*
}

\author{
Fernando Franklin GRINSTEIN**
}

\begin{abstract}
Large Eddy Simulation (LES) of shear flows driven by Kelvin-Helmholtz instabilities such as mixing layers, wakes, and jets is of great interest because of their crucial role in many practical applications. The Monotone Integrated LES (MILES) approach is motivated here for these studies, and the basic components involved in a typical MILES jet model are described. Examples from MILES jet studies are used to address major aspects of transition to turbulence from laminar conditions at the nozzle exit including, the occurrence of global instabilities, complex three-dimensional vorticity geometries, and their impact on jet entrainment. Quantitative analysis of the small-scale features of the transitioning simulated jets is presented, and convergence issues are addressed in this context.
\end{abstract}

Key Words: Jets, Coherent Structures, Transition, MILES, Entrainment, Instabilities

\section{Miles Approach}

Simulation of jet flows is based on the numerical solution of the Navier-Stokes (NS) or Euler (EU) equations with appropriate boundary conditions. The important issues that need to be addressed relate to the appropriate modeling of: 1) the required open boundary conditions for flows developing in both space and time, and, 2) the unresolved subgrid scale (SGS) flow features. Supergrid modeling with appropriate boundary conditions is required to ensure that the presence of computational boundaries adequately bounds the computational domain without polluting the solution in a significant way, while modeling effects of virtual flow events (outside of the domain) emulating conditions in the laboratory flows. High Reynolds' number $(R e)$ turbulent flows contain a broad range of scales of length and time. The largest length scales are related to the flow configuration geometry and boundary conditions whereas it is principally at the smallest length scales that energy is dissipated by molecular viscosity. Subgrid scale models are needed to ensure the accurate computation of the largest (grid-scale) resolved motions responsible for the primary jet transport and entrainment.

Simulations that capture all the relevant length scales of motion though numerical solution of the NS equations are termed direct numerical simulation (DNS). DNS is prohibitively expensive, now and for the foreseeable fu-

\footnotetext{
* Received 24th February, 2006 (No. 06-5037)

** Los Alamos National Laboratory, MS-B259, Los Alamos, NM 87545, USA. E-mail: fgrinstein@lanl.gov
}

ture, for most practical flows of moderate to high $R e$. Large eddy simulation (LES) ${ }^{(1)}$ is an effective approach that is intermediate in computational complexity between the Reynolds-averaged Navier Stokes (RANS) strategies and DNS, and addresses some of the shortcomings of RANS at a reasonable cost. The main assumptions of LES are: (i) that the transport of momentum, energy, and passive scalars is largely governed by the large length scale unsteady features that can be resolved in space and time; (ii) the smaller length scales are more universal in their behavior, and their effect on the large scales (e.g., in dissipating energy) can be represented by using suitable Subgrid Scale (SGS) models.

Experimental investigations have shown that largescale vortical coherent structures (CS) dominate the entrainment and mixing processes in free shear flows ${ }^{(2),(3)}$. By design, LES approaches focus on modeling largescale-driven features of the flow, and are thus ideally suited to model such flows. Conventional LES involves low-pass filtering of the NS equations followed by explicit SGS modeling ${ }^{(1)}$. LES seeks to resolve most of the entrainment-dominating CS by choosing the cutoff wavelength within the inertial subrange. Many different approaches have been developed for the construction of SGS models ${ }^{(1)}$. It is essential to recognize that in the absence of a universal theory of turbulence, the construction of SGS models is unavoidably pragmatic, and primarily based on the rational use of empirical information. The main challenge is to appropriately emulate the fluid dynamics near the cut-off, to ensure that proper interactions between re- 
solved and SGS scales are simulated.

The idea that a suitable SGS model might be implicitly provided by discretization with a particular class of high resolution numerical methods led to proposing the MILES approach $^{(4),(5)}$. Early MILES studies were able to reproduce the large-scale features of the flow observed in the laboratory experiments, e.g., the asymmetric entrainment $^{(6)}$ the distribution of merging locations and spreading rate of the mixing layers ${ }^{(7),(8)}$ and the base-pressure and vortex-shedding dynamics in bluff-body near-wakes ${ }^{(9)}$. Important milestones to specially mention here were the first demonstration of physical global instabilities in free mixing layers ${ }^{(10)}$, and the first three-dimensional MILES of free jets transitioning from laminar initial conditions ${ }^{(11)}$ - some of which are reviewed in what follows. Later theoretical studies $^{(12)-(14)}$ showed that certain class of fluxlimiting algorithms with dissipative leading order terms provide appropriate built-in (implicit) SGS models of a mixed tensorial (generalized) eddy-viscosity type. MILES based on using Flux-Corrected Transport (FCT) algorithms have been extensively used in the study of free shear flow phenomena to investigate the vortex dynamics and mechanisms of transition to turbulence in moderatelyhigh $R e$ planar mixing layers ${ }^{(6),(10)}$, planar wakes ${ }^{(15),(16)}$, in jets emerging from round ${ }^{(17),(18)}$ and rectangular ${ }^{(19)-(22)}$ nozzles, as well as in swirling jet flows ${ }^{(23)}$. MILES has also been extensively used to investigate convectivelydominated chemically reacting jets (see, e.g., Refs. (22), (24), (25) and references therein).

\section{Miles Jet Model}

In the (subsonic) compressible free shear-flow simulations discussed below, complex vortex dynamics and its associated acoustical production must be distinctly captured, and spurious boundary condition effects need to be avoided (or at the very least, clearly identified and controlled). MILES is based on solving the time-dependent, compressible flow conservation equations for mass, energy, and momentum density and chemical species (when appropriate) with an ideal gas equation of state, and using 4th order FCT finite volume algorithms at the convection stage. The simulations are based on either the unfiltered EU equations - denoted here as the nominallyinviscid case, MILES-EU, or the NS equations without (MILES) or with a Smagorinsky-type viscosity (MILESSMG). The simulations are based on splitting the integrations for streamwise convection, cross-stream convection and other local processes (e.g., viscosity, thermal conduction, molecular diffusion and finite rate chemistry, as appropriate). Convective spatial integrations are performed utilizing 1D FCT for the streamwise direction, 2D FCT on cross-stream planes, and 3D central differences otherwise. Time-integrations are implemented using a 2 nd order predictor-corrector scheme (see discretization model discussion in Ref. (12).

Open boundary conditions are imposed at open boundaries in the streamwise direction (inflow and outflow), and free-stream flow conditions are imposed at the cross-stream directions. Inflow boundary conditions specify the guard-cell values for the mass density and velocities. The inflow guard-cell pressures are obtained as solutions of a one-sided finite-difference expression based on the one-dimensional, unsteady, inviscid pressure equation $^{(26)}$. A non-reflecting one-dimensional boundary condition with or without explicit asymptotic relaxation to ambient conditions on the pressure is specified at the downstream (outflow boundary), where the additional numerical conditions required for closure of the discretized equations are introduced by requiring that the mass and momentum densities be advected with the local streamwise velocity at the boundary ${ }^{(26)}$. Structured cartesian computational grids used were held fixed in time, and used evenly spaced cells in the shear flow region of interest. Geometrical stretching in the cross-stream and downstream directions outside of the latter region was frequently used to implement the open boundary conditions there. More specific details can be found in the cited references.

\section{Transition to Turbulence}

Crucial to the shear flow development transitioning from laminar conditions to turbulence, is the rate at which fluid elements from the free streams become entangled or mixed as they join at the mixing layers. This information is given by the entrainment rates, which define the rate of propagation of the interface between rotational and irrotational fluid. Viscous diffusion of vorticity acting primarily at the smallest scales of motion where the gradients are the largest, acts to propagate vorticity into the irrotational fluid. However, the production of this small scale vorticity is largely controlled by the straining provided by much larger scales of motion. Thus the entrainment rate is controlled by the speed at which the interface contortions with the largest scales move into the surrounding fluid ${ }^{(27)}$. These controlling large scale vortices tend to be coherent and easily recognizable features, hence their name CS. Control of the shear flow development in the practical applications is strongly dependent on understanding the dynamics and topology of CS; in particular, how their properties can be affected through control of the formation, interaction, merging, and breakdown of CS. Selected examples from the MILES jet studies are used below to demonstrate their important contributions in representative applications.

\subsection{Global instabilities}

Characterizing the local nature of free shear-flow instabilities and their global nonlinear development in space and time is of fundamental importance for practical shear flow control. An important question is whether a free mix- 
ing layer can be globally unstable with the self-excitation upstream induced by pressure disturbances generated via finite amplitude fluid accelerations downstream. From the point of view of linear inviscid stability analysis, the spatially evolving subsonic mixing layer is convectively unstable with respect to vortical fluctuations ${ }^{(28)}$. As a consequence, turbulent mixing layers are expected to be driven by environmental disturbances ${ }^{(29)}-$ except in rare configurations with global-absolute instabilities - and selfexcitation effects in free shear flows are not generally expected to be significant.

Mechanisms triggering instabilities and transition to turbulence in free shear flows (e.g., vortex roll-up and merging) are: disturbances in the free streams, disturbances due to boundary layers, wakes, small recirculation zones, or acoustic environmental disturbances, and disturbances fed back from downstream events in the flow. Isolating these mechanisms is difficult in the laboratory studies because turbulence in free streams and boundary layers cannot be eliminated. Numerical simulations of spatially evolving shear flows can eliminate the first two disturbances, and can address the third through careful control of the prescribed boundary conditions. Grinstein et al. ${ }^{(10)}$ successfully addressed these questions with FCT-based MILES-EU of a spatially evolving mixing layer in which effects of the boundaries could be neglected. The studies gave direct evidence for the existence of a global instability mechanism for self-excitation of the mixing layer, and thus for self-sustaining instabilities, due to upstream feedback from the fluid accelerations originated by the vortex roll-ups and vortex pairings downstream (Fig. 1). Later studies demonstrated similar self-excited global instabilities in supersonic jets, based on upstream feedback mechanisms acting on the subsonic outer jet regions ${ }^{(30)}$.

Figure 1 (b) shows time series of velocity and pressure at the center of the shear layer $(y=0)$, at selected streamwise locations: traces 1-7 marked on the vertical axis are cross-stream velocity $u$ and trace "0" is the crossstream pressure derivative just ahead of the inflow boundary. The streamwise stations corresponding to the traces indicated in Fig. 1(a), and each individual trace value is scaled by its maximum value. Trace 7 at the top of the figure was recorded near the middle of the computational domain. Upstream acoustic rays are marked as solid straight lines from the upper left to the lower right (e.g., rays $\Gamma_{1}$, $\Gamma_{2}, \Gamma_{3}$ ), and (dashed) rays of fluid convection go from the lower left toward the upper right. The dashed rays are used to examine the convective growth of disturbances, whereas the solid, upstream propagating rays are used to assess the possible role of fluid acceleration events downstream in generating the fluctuations observed near the inflow. The peaks in the velocity traces correspond to the passage of vortex rolls. The first such peak on traces 1-4 corresponds to the roll-up of the vortex on the left of the

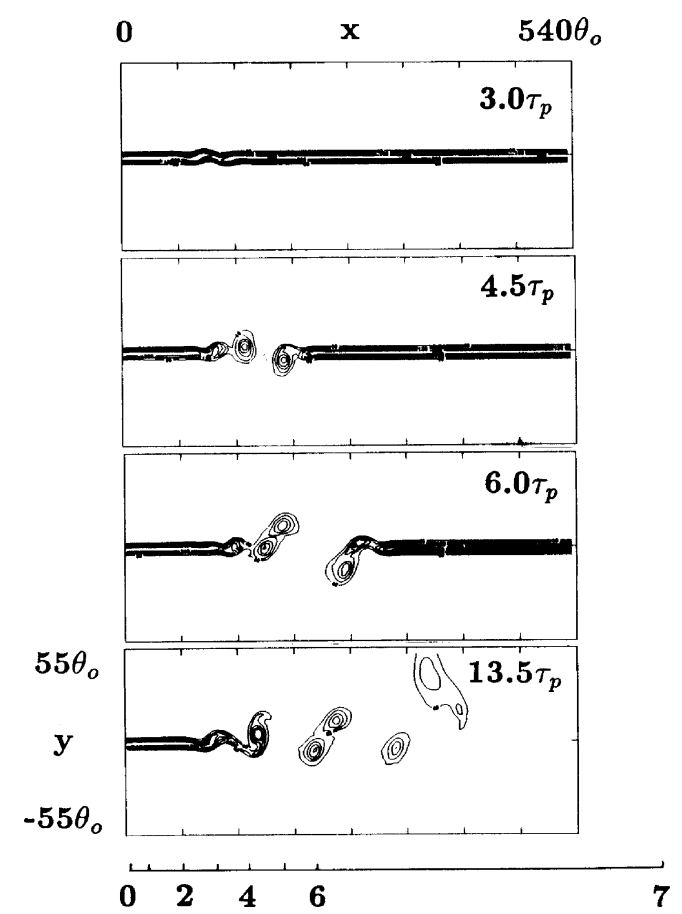

(a) Global instabilities in the convectively unstable planar mixing layer (from Ref. (10))

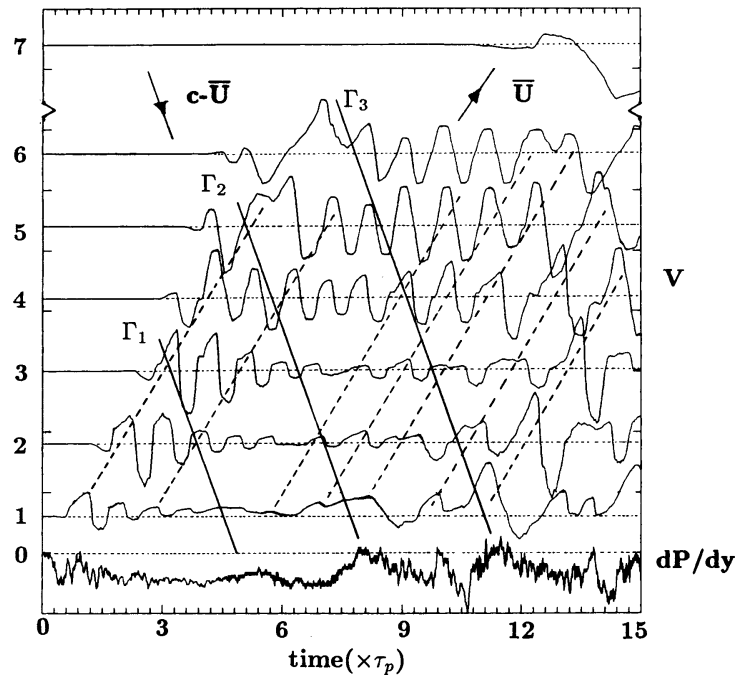

(b) Time series of transverse velocity and pressure derivative at streamwise locations in Fig. 1 (a)

Fig. 1 The self excited subsonic mixing layer

initial vortex pair shown in the upper panels of Fig. 1 (a). The peak velocity fluctuation level in the center of the shear layer increases very fast as we move downstream and saturates in the neighborhood of trace 4 , where the peaks associated with vortex roll-ups appear quite regularly. See Ref. (10) for further details.

The idea of a feedback mechanism through which downstream flow events can influence the upstream flow was first proposed by Dimotakis \& Brown ${ }^{(31)}$ to explain unusually long autocorrelation times of the streamwise velocity fluctuations observed in their planar shear flow 


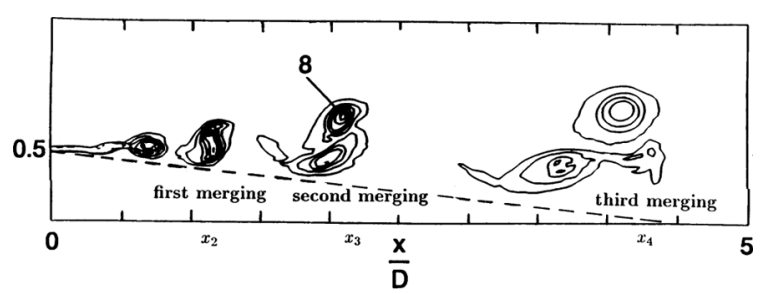

(a) Instantaneous contours of azimuthal vorticity for a simulated $M=0.57$ round jet (from Ref.(17)), depicting vortex ring merging events

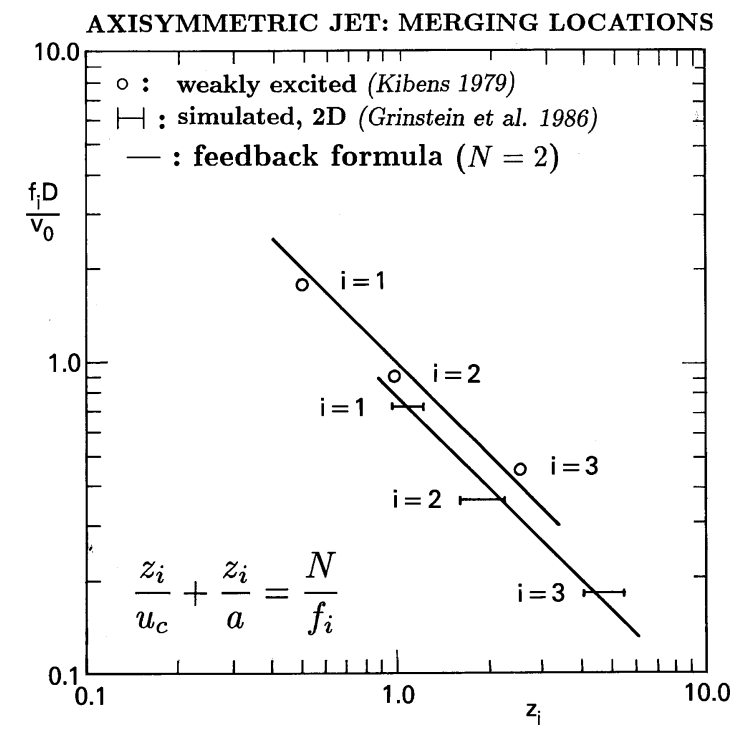

(b) Distribution of vortex ring merging locations in simulated and laboratory round jets (from Ref. (16))

Fig. 2 Jet self organization

experiments. Subsequently, Laufer \& Monkewitz ${ }^{(32)}$ observed that the unstable shear layer close to the nozzle of a jet, was modulated with a low frequency corresponding to the passage frequency of the large scale structures at the end of the potential core. In order to explain the observed jet self-organization, a feedback mechanism for free and forced shear flows was proposed in which the cross-stream perturbations required for the vortex pairing process are provided by the pressure field induced by the downstream (already paired) vortices ${ }^{(32),(33)}$. The feedback loop from the trailing edge to the merging location involves the unstable vortex roll-ups convecting downstream and pressure waves from these changing, predominantly rotational flows, propagating upstream. The resulting feedback formula implied by modeling this mechanism describes fairly well the distribution of merging locations found in weakly excited experimental jets ${ }^{(32),(33)}$ and was also captured in the MILES studies (Fig. 2) ${ }^{(7),(8)}$.

Figure 2 (a) shows typical instantaneous contours of the azimuthal vorticity from the MILES studies of axisymmetric jets in Ref. (8). The initial axisymmetric vorticity sheet rolls up into vortex rings, which move downstream, interact with each other, and thereby spread the vorticity until the central, potential core of the jet disappears. For the particular jet initial conditions considered, the phase shown in the figure depicts a vortex-ring resulting from a first merging at $x_{2}$, two (merged) vortices aligned vertically at $x_{3}$ undergoing a second merging, and a third such vortex-ring-merging process at $x_{4}$ involving vortices resulting from a second merging. Through essentially inviscid vortex pairing interactions, the transverse crosssection of the ring structures nearly doubles at each merging. The vortex merging locations tend to be distributed in fairly regular patterns (Fig. 2 (b)), which can be fit by the so-called feedback formula, involving downstream convection velocity $u_{c}$ and upstream acoustic propagation velocity " $a$ ". Extensive experimental investigations were unable to conclusively establish the existence of this feedback loop for laboratory free jets due to so-called unavoidable facility-dependent background disturbances ${ }^{(34)}$ - reflecting the fact that different facility boundary conditions prescribe different jet flows. The feedback loop was first isolated and demonstrated in the MILES studies of global instabilities described above (Fig. 1).

A key computational capability used in the studies in Refs. (7), (8) and (10) was the ability to isolate the generation and propagation of acoustical disturbances correlated with the large-scale vortex dynamics. Relevant features accurately captured with MILES included the quadrupole pattern of acoustic production associated with vortices, the significantly more intense dilatation and pressure fluctuations associated with vortex pairing, as well as the very low fluctuation levels involved (e.g., four orders of magnitude smaller than ambient values).

\subsection{Complex vortex dynamics}

Mixing between free streams occurs in two stages, an initial stage of bringing relatively large amounts of the fluids together (large-scale stirring), and a second stage promoted by the small-scale velocity fluctuations which accelerate mixing at the molecular level. Large scale twodimensional spanwise rollers (in plane mixing layers or wakes), or vortex rings (in round jets such as discussed above) and their interactions play a crucial role in controlling transitional shear-layer growth and entrainment at moderately-high $R e$. For higher $R e$, and/or when the azimuthal or spanwise symmetry is broken or not present, three-dimensionality becomes the crucially important feature. Now, additional CS such as streamwise or longitudinal (e.g., rib, hairpin) vortices, and mechanisms such as self-induction, vortex stretching, vorticity production due to baroclinicity and expansion as well as vortex reconnection become major fluid-dynamical processes involved in the transition from laminar to turbulent regimes.

Practical jet entrainment control involves manipulating the natural development of CS and their breakdown into turbulence to enhance three-dimensionality and thus mixing. Popular passive control approaches are based 
on geometrical modifications of the jet nozzle which directly alter the flow development downstream relative to using a conventional circular nozzle; research on flow control with non-circular jets were reviewed by Gutmark \& Grinstein $^{(35)}$. Rectangular jet configurations are of particular interest because they offer passively-improved mixing at both ends of the spectrum: enhanced large scale entrainment due to axis-switching, and enhanced small scale mixing near corner regions and farther downstream - due to faster breakdown of vortex ring coherence and hence faster transition to turbulence.

Rectangular jet MILES studies (e.g., Refs. (21) and (22)) with aspect ratio $A R=1-4$, were carried out in various non-reactive and reactive regimes. As in the case of simulated round jets discussed above, for computational efficiency, the focus was on initially laminar jets, developing from thin rectangular vortex sheets (with slightly rounded-off corner regions here), and uniform momentum thickness $\theta$ enforced in terms of appropriate top-hat velocity profiles. Characteristic velocity scales for the rectangular jet simulations were $U_{j}$ and the peak $r m s$ velocity fluctuation value $u^{\prime}$, where $U_{j}$ denotes the free-stream jet velocity. The gas jets investigated were assumed to emerge into a quiescent background with Mach number $M=0.6$. Characteristic length scales are the initial shear layer thickness, the jet equivalent diameter $D_{e}$ and the Taylor microscale $L_{T}=L / \sqrt{\left(R e_{T}\right)}$, where $R e_{T}=u^{\prime} L / v$, and the integral scale $L$ is the full core width of the initial vortex rings (size of the largest eddies in the flow). Typical $R e$ for the nominally inviscid jets involved $R e=U_{j} D_{e} / v>$ $78000\left(R e_{T}>90\right)$ - based on estimated upper bounds for the effective numerical viscosity of the FCT algorithm ${ }^{(36)}$ or comparisons with DNS turbulence data ${ }^{(13),(22)}$; typical DNS studies involved $R e=3200$, based on air viscosity at STP.

The jet simulations demonstrated the dynamical vorticity geometries characterizing the near jet, involving: i) self-deforming vortex rings, ii) interacting ring and braid (rib) vortices, and, iii) a more disorganized flow regime in the far jet downstream, where the rotational fluid volume is occupied by a relatively weak vorticity background with strong, slender tube-like filament vortices filling a small fraction of the domain. Figure 3 is used to highlight the characteristic observed differences as a function of $A R$ based on representative instantaneous volume visualizations. Two qualitatively different ring-rib coupling geometries are shown: jets with $A R \geq 2$ are characterized by single ribs aligned with corner regions - in contrast with pairs of counter rotating ribs aligned with the corners for square $(A R=1)$ jets. Other distinct topological features are associated with the occurrence of vortex ring splittings due to reconnection which are observed for the larger values $A R$. Figure 4 (a) illustrates characteristic axis-switching and bifurcation phenomena for $A R=4$

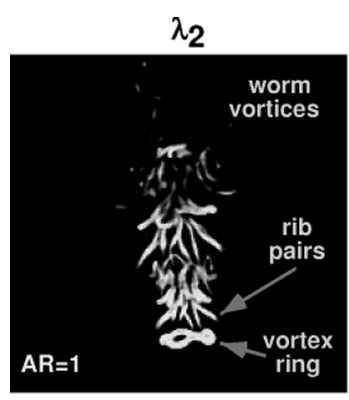

streamwise vorticity
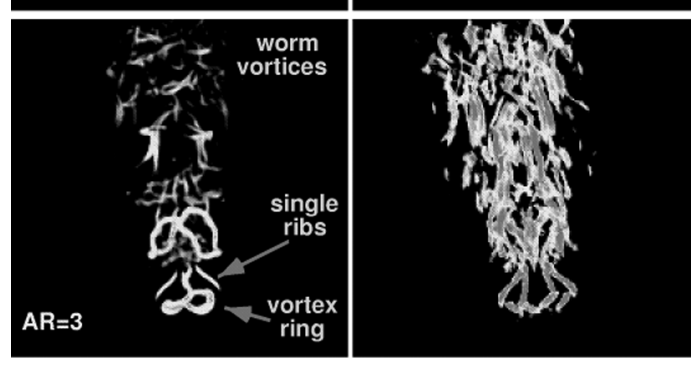

Fig. 3 Comparative vortex geometries for rectangular jets with varying nozzle aspect ratio (from Ref. (22))
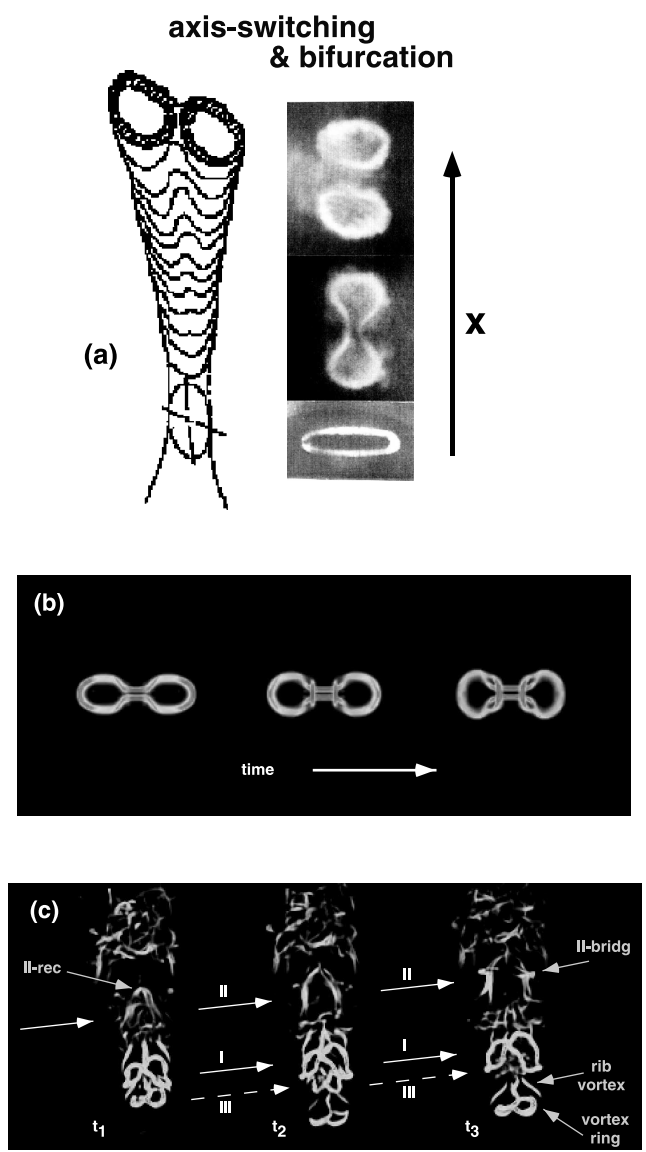

Fig. 4 Vortex splitting in rectangular jets. (a) laboratory studies $^{(37)}$; (b) and (c) MILES ${ }^{(19),(22)}$

from visualizations of laboratory elliptical jets subject to strong excitation at the preferred mode ${ }^{(37)}$. We compare it to the carefully developed simulations results (Fig. 4 (b) and (c)) designed to address unresolved issues in vortex dynamics. Detailed key aspects of the bifurcation dynam- 
ics - namely, reconnection, bridging, and threading were conjectured in Ref.(37) but not be captured in the laboratory studies, and were first demonstrated by the simulations $^{(19),(22)}$.

In terms of characteristic ring-rib coupling geometry, significantly larger jet spreading and streamwise vorticity $\left(\omega_{1}\right)$ production in the jet for $A R=1$ are shown in Fig. 3 reflecting on the appearance of rib-pairs aligned with corner regions - rather than single ribs for $A R=3$. On the other hand, also associated with the larger near-jet $\omega_{1}$ production for $A R=1$, the vortex rings tend to be more unstable azimuthally and break down closer to the jet exit. As a consequence, Fig. 3 presents larger jet spreading for $A R=3$ further downstream (say for $x>6 D_{e}$ ), where better entrainment properties are suggested by the more intense distributions of streamwise vorticity (also more intense for $A R=3$ than for $A R=2$ ). Furthermore, vortexself-deformation and axis-switching can occur (and promote mixing better) closer to the jet exit for the $A R=1$ case due to significantly smaller characteristic vortex-ring axis-switching times - i.e. typically half-as-large and less than one-third-as-large, respectively, compared with those for $A R=2$ and $A R=3$ (e.g., Ref. (19)). Additional effects of initial jet condition specifics previously addressed $^{(20),(22),(35)}$, include examining the role of momentum thickness azimuthal non-uniformities, nozzle geometry and nature (e.g., contraction vs. pipe or orifice nozzles) and their impact on axis-switching, entrainment and combustion. Extensive quantitative comparisons between simulated and laboratory jet were also reported in the cited references.

Thus, there are different interesting possibilities for how the jet flow develops, depending on the particular jet initial conditions and specific type of unsteady vortex interactions initiated, the nozzle geometry and modifications introduced at the jet exit. This is of interest in the context of improving the mixing of a jet (or plume) with its surroundings in many practical applications. Taking advantage of these flow control possibilities is of interest to improve the mixing of a jet, or plume, with its surroundings in practical applications demanding, e.g., enhanced combustion between injected fuel and background oxidizer, rapid initial mixing and submergence of effluent fluid, less intense jet noise radiation, or reduced infrared plume signature. The crucial feature to control here is the jet entrainment rate - the rate at which fluid from the jet becomes entangled or mixed with its surroundings, determined by the characteristic large-scale vortex dynamics. MILES can be particularly useful in this research. Because of the tensorial nature of its implicit SGS model, and the inherently-low numerical diffusion involved, it offers an overall-effective computational alternative to conventional SGS models when seeking refined LES for inhomogeneous turbulent flows as required in this context.

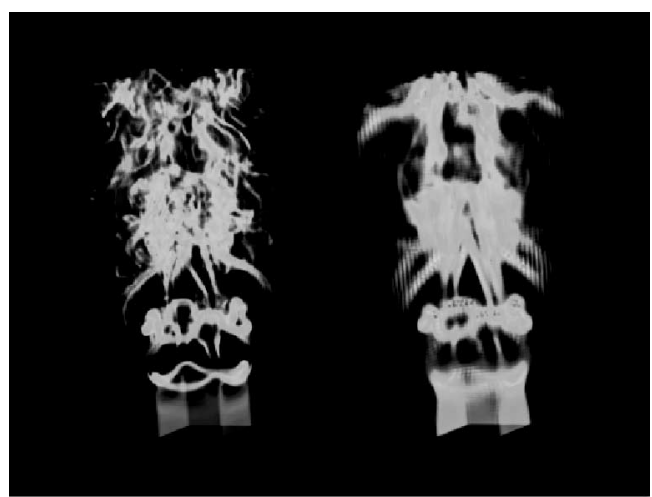

Fig. 5 Instantaneous volume visualizations of the vorticity magnitude in a square jet (twice as resolved on the left); time is the same on both frames ${ }^{(21),(22)}$

\subsection{Small scale emulation}

Figure 5 shows instantaneous visualizations of a developed square jet from MILES-EU studies ${ }^{(21),(22)}$. To facilitate the analysis of the results, the jet flow was organized using weak forcing with (single) Strouhal frequency $S t=f D_{e} / U_{j}=0.55$, chosen within the range of observed laboratory preferred (large scale) jet frequencies, and rms level $2.5 \%$ of $U_{j}$. Forcing amplitude was chosen to be low enough to ensure good agreement of the initial shear-layer growth rate of the simulated jets with those of comparable unforced laboratory orifice jets with nearly-laminar initial conditions. The figure compares unfiltered data at selected representative times from simulations carried out with identical initial and boundary conditions in grids denoted $\mathrm{F}$ and $\mathrm{C}$, with nominal smallest cell spacings $\Delta$ and $2 \Delta$ (and corresponding timesteps imposed by the fixed Courant number constraint), and estimated $R e$ (based on $U_{j}, D_{e}$, and effective algorithm viscosity), $R e>22000$, and $R e>78000$, respectively. The smallest grid size used was $\Delta=D / 42$, where $D$ is the length of the minor side of the rectangular nozzle. These comparisons and further analysis below, are used to examine dependence on spatial resolution (effective $R e$ ) of the simulations as well as issues of MILES convergence.

Figure 5 depicts the square vortex sheet rolling up into vortex rings near the inflow, self-induced distortion of the nominally-square vortex rings, formation of hairpin (braid) vortices between distorted rings, strong coupling of these vortices into ring-hairpin bundles, and merging of the vortex bundles. Vortex interactions and azimuthal instabilities lead to more contorted vortices; vortex stretching, kinking, and reconnection lead to their breakdown, and to a more disorganized flow regime farther downstream, characterized by elongated vortices resembling those typical of fully developed turbulent flows (e.g., Refs. (38) and (39)). The figures show quite good visual agreement on the large-scale dynamics of ring and hairpin vortices near the jet exit, but their faster coherence 
breakdown downstream is apparent with increasing resolution (effective $R e$ ), as finer dynamical features can contribute and affect the larger scales. As a consequence, observations are unavoidably affected by the simulated process ! Very important to note here is that this is not special from MILES, it is actually inherent in the LES approach itself, where we are always constrained to simulate a flow with smallest characteristic resolved scale determined by a resolution cutoff wavelength prescribed by a spatial-filtering process. This is irrespective of whether the characteristic filter-length is (explicitly) prescribed or not independently of grid resolution: although some LES methods can be claimed to be "grid-independent" they are always filter-length dependent !

In what follows, we focus on making quantitative statements on the trends of the population of the smallscales vortices in the downstream portion of the simulated jets, where the flow is characterized by thinner filament vortices similar to those observed in fully developed turbulent flows. We compare the features of the simulated turbulence to which the simulated flow transitions from laminar initial conditions with what is regarded as its established metrics. Spectral analysis concentrating on the latter portion of the simulated developed jets, can be used to investigate the small-scale jet behavior captured by the simulations. Analysis is based on the instantaneous velocity databases for the grid resolutions considered on (uniformly spaced) downstream subvolumes. These volumes include only the appropriate downstream portions of the jets chosen symmetrically around the centerline ${ }^{(12),(13),(21),(22)}$.

Figure 6 (a) shows time-averaged plots of the turbulent kinetic energy spectra in the case $A R=1$. In each case, spatial FFT analysis is based on datasets for 40 successive times separated by a time interval $0.1 / f$, on $140^{3}$ and $70^{3}$ downstream grid sub-volumes, for the two resolutions. The largest wavenumber for which spectral amplitudes are plotted corresponds to a wavelength of four computational cells; the spectra depict short simulated inertial subranges consistent with the $k^{-5 / 3}$ inviscid subrange of the Kolmogorov K41 theory, and a longer inertial range for higher resolution case. Figure 6 (b) shows spectra as function of $A R$ for intermediate resolution (1.5D), and based on $110^{3}$ downstream subvolumes; the figure suggests the experimentally observed shallower slope (than the $k^{-5 / 3}$ slope) in the near-dissipation region (e.g., Ref. (40)). This was also noted in turbulent decay MILES studies using the 4th order Piecewise Parabolic Method (PPM) $)^{(39)}$. It is a characteristic feature of high (but finite) Re solutions of the NS equations - effectively emulated through MILESEU. This can be interpreted as indicating that in spite of relying on inherently dissipative-type of SGS modeling, MILES can implicitly incorporate some degree of desirable backscatter dependent on the existence of a simulated

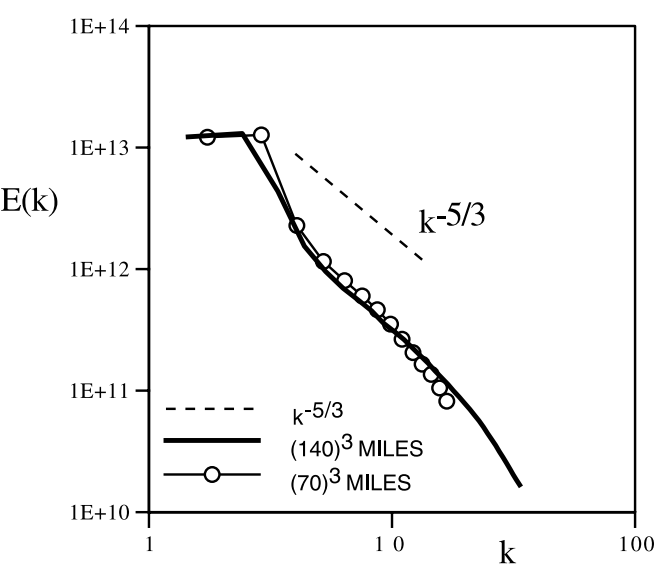

(a) Mean kinetic energy spectra for the jets in Fig. 5

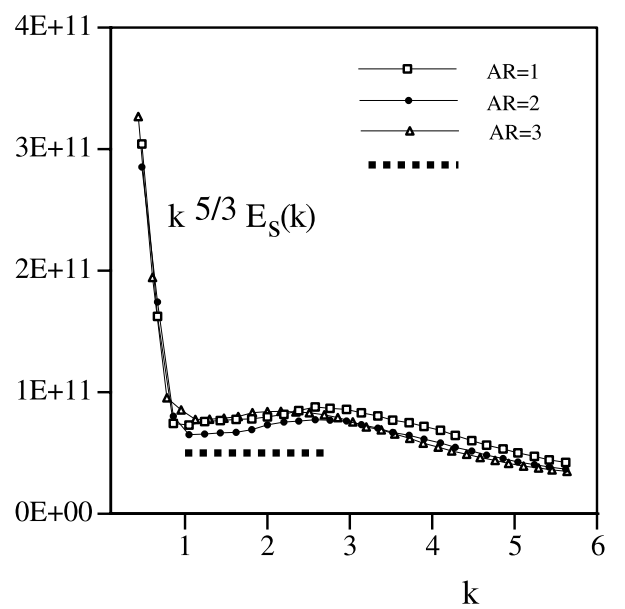

(b) Compensated mean spectra for the jets in Fig. 5

Fig. 6 Spectral analysis of simulated data (from Ref. (13))

dissipation region - but fairly independent of its precise nature. The simulated inertial range is followed by faster decay of the amplitudes due to the FCT dissipation for wavelengths $\lambda<10 \Delta$. This limiting length-scale corresponds to approximately twice the smallest characteristic (full-width) cross-sectional length scales of the elongated vortex tubes in the transitional region of the jet ${ }^{(21)}$.

The distribution of vorticity intensities can be examined based on the cumulative distribution function (CDF), defined as the volume fraction occupied by vorticity magnitude values above a given threshold level. Figure 7 focuses again on the jet with $A R=1$, showing CDF's of the normalized vorticity magnitude $\omega$ for $\omega \geq 1$ based on downstream subvolume data for the two extreme grid resolutions F \& C, superimposed to CDF's based on DNS turbulence data by Jiménez et al. ${ }^{(38)}$ The vorticity magnitude is scaled with its rms value $\omega^{\prime}$ for each jet subvolume data. The figure shows good agreement of the finest resolved grid data with the DNS data even for fractional volumes of less than $1 \%$, while differences between DNS and the coarsest grid data are significant much before, for fractional volumes between $10 \%$ and $1 \%$. The structure of the resolved vorticity implies that high intensity regions 


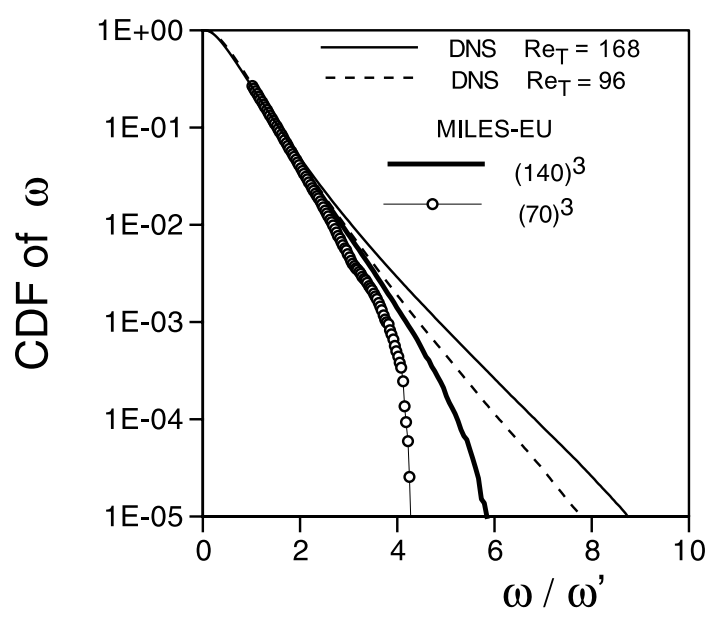

Fig. 7 Vorticity distributions for the jets in Fig. 5

tend to be organized mostly in elongated tubes. Because "worm" vortices typically involve fractional volumes of order $1 \%$ or less $^{(38)}$, of these two cases, only the finer grid simulation resolves these vortices, and this is supported by the volume visualizations of Fig. 5 .

The possible dependence of the resolved small-scale jet features discussed above on the specifics of the SGS modeling were assessed based on the Euler and NS equations with and without SMG viscosity ${ }^{(12),(13)}$. The simulations depicted virtually identical initial (large scale) jet dynamics; globally similar but distinctly different local features were apparent as the flow regime becomes disorganized and dominated by the presence of thin elongated vortices, but with very similar velocity-fluctuation spectra for all three MILES approaches, indicating: (i) somewhat smaller amplitudes for lower wavenumbers associated with MILES and MILES-SMG, depicting viscous damping of resolved features; (ii) essentially coincident high-wavenumber amplitudes - reflecting the unresolved small-scale viscous effects; (iii) captured inertial subrange. The distributions of vorticity magnitude, rateof-strain, and stretching turned out to be correspondingly very similar for the three types of MILES.

\section{Outlook}

MILES seeks to emulate the flow features in the highwave-number end of the inertial subrange region of turbulent flows - characterized by thin filaments of intense vorticity embedded in a background of weak vorticity. By focusing on the inviscid inertial-range dynamics and on regularization of the under-resolved flow, the approach follows up very naturally on the historical precedent of using this kind of numerical schemes for shock capturing.

Tests in fundamental applications ranging from canonical to complex flows (see recent surveys in Refs. (41) - (43)) indicate that - while computationally simpler and more economical - MILES is competitive with the conventional LES approaches. Because of the tenso- rial nature of its implicit SGS model, and the inherentlylow numerical diffusion involved, it offers an overalleffective computational alternative to conventional SGS models when seeking refined LES for inhomogeneous turbulent flows.

\section{References}

(1) Sagaut, P., Large Eddy Simulation for Incompressible Flows, 3rd Edition, (2005), Springer, New York.

(2) Brown, G. and Roshko, A., On Density Effects and Large Structure in Turbulent Mixing Layers, J. Fluid Mech., Vol.64 (1974), pp.775-816.

( 3 ) Hussain, A.K.M.F., Coherent Structures and Turbulence, J. Fluid Mech., Vol.173 (1986), pp.303-356.

( 4 ) Boris, J.P., On Large Eddy Simulation Using Subgrid Turbulence Models, Whither Turbulence? Turbulence at the Crossroads, Edited by Lumley, J.L., (1990), pp.344-353, Springer, NY.

( 5 ) Boris J.P., Grinstein, F.F., Oran, E.S. and Kolbe, R.J., New Insights into Large Eddy Simulation, Fluid Dyn. Res., Vol.10 (1992), pp.199-228.

( 6 ) Grinstein, F.F., Oran, E.S. and Boris, J.P., Numerical Simulations of Asymmetric Mixing in Planar Shear Flows, J. Fluid Mech., Vol.165 (1986), pp.201-220.

( 7 ) Grinstein, F.F., Oran, E.S. and Boris, J.P., Direct Numerical Simulation of Axisymmetric Jets, AIAA J., Vol.25 (1987), pp.92-98.

( 8 ) Grinstein, F.F., Hussain, F. and Oran, E.S., Vortex-Ring Dynamics in a Transitional Subsonic Free Jet. A Numerical Study, Europ. J. Mech., B/Fluids, Vol.9, No.6 (1986), pp.499-525.

(9) Grinstein, F.F., Boris, J.P. and Griffin, O.M., Passive Pressure-Drag Control in a Plane Wake, AIAA J., Vol.29, No.9 (1991), pp.1436-1442.

(10) Grinstein, F.F., Oran, E.S. and Boris, J.P., Pressure Field, Feedback and Global Instabilities of Subsonic Spatially Developing Mixing Layers, Phys. Fluids, Vol.A3, No.10 (1991), pp.2401-2409.

(11) Grinstein, F.F. and DeVore, C.R., Coherent Structure Dynamics in Spatially Developing Square Jets, AIAA Paper 92-3441, (1992).

(12) Fureby, C. and Grinstein, F.F., Monotonically Integrated Large Eddy Simulation of Free Shear Flows, AIAA J., Vol.37, No.5 (1999), pp.544-556.

(13) Fureby, C. and Grinstein, F.F., Large Eddy Simulation of High Reynolds Number Free \& Wall Bounded Flows, J. Comp. Phys., Vol.181 (2002), pp.68-97.

(14) Grinstein, F.F., Fureby, C. and DeVore, C.R., On MILES Based on Flux-Limiting Algorithms, Int. J. Numer. Meth. Fluids, Vol.47 (2005), pp.1043-1051.

(15) Grinstein, F.F., Hussain, F. and Boris, J.P., Dynamics and Topology of Coherent Structures in a Plane Wake, Advances in Turbulence 3, Edited by Johansson, A.V. and Alfredsson, P.H., (1991), pp.34-41, Springer, Berlin, Heidelberg.

(16) Jeong, J., Grinstein, F.F. and Hussain, F., Eduction of Coherent Structures in a Numerically Simulated Plane Wake, Appl. Scientific Research, Vol.53 (1994).

(17) Grinstein, F.F., Glauser, M.N. and George, W.K., Vorticity in Jets, Fluid Vortices, Edited by Green, S.I., 
(1995), p.65, Kluwer, Dordrecht.

(18) Grinstein, F.F., Gutmark, E.J., Parr, T.P., Hanson-Parr, D.M. and Obeysekare, U., Streamwise and Spanwise Vortex Interaction in an Axisymmetric Jet. A Computational and Experimental Study, Phys. Fluids, Vol.8, No.6 (1996), pp.1515-1524.

(19) Grinstein, F.F., Self-Induced Vortex Ring Dynamics in Subsonic Rectangular Jets, Phys. Fluids, Vol.7, No.10 (1995), pp.2519-2521.

(20) Grinstein, F.F., Gutmark, E. and Parr, T.P., Near-Field Dynamics of Subsonic, Free Square Jets. A Computational and Experimental Study, Phys. Fluids, Vol.7, No.6 (1995), pp.1483-1497.

(21) Grinstein, F.F. and DeVore, C.R., Dynamics of Coherent Structures and Transition to Turbulence in Free Square Jets, Phys. Fluids, Vol.8, No.5 (1996), pp.12371251.

(22) Grinstein, F.F., Vortex Dynamics and Entrainment in Regular Free Jets, J. Fluid Mech., Vol.437 (2001), pp.69-101.

(23) Grinstein, F.F., Young, T.R., Gutmark, E.J., Li, G., Hsiao, G. and Mongia, H.C., Flow Dynamics in a Swirl Combustor, J. Turbulence, Vol.3, No.030 (2002), pp.119.

(24) Grinstein, F.F., Vortex Dynamics, Entrainment, and Non-Premixed Combustion in Rectangular Jets, Chapter 13, Advances in Chemical Propulsion, Edited by Roy, G., (2002), pp.215-230, CRC Press.

(25) Grinstein, F.F. and Fureby, C., LES Studies of the Flow in a Swirl Gas Combustor, Proceedings Combustion Institute, Vol.30 (2005), pp.1791-1798.

(26) Grinstein, F.F., Open Boundary Conditions in the Simulation of Subsonic Turbulent Shear Flows, J. Comp. Phys., Vol.115 (1994), pp.43-55.

(27) Tennekes, H. and Lumley, J.L., A First Course in Turbulence, (1972), MIT Press, Cambridge, MA.

(28) Huerre, P. and Monkewitz, P.A., Local and Global Instabilities in Spatially Developing Flows, Annual Rev. Fluid Mech., Vol.22 (1990), pp.473-537.

(29) Morkovin, M., Recent Insights into Instability and Transition to Turbulence in Open-Flow Systems, ICASE Report 88-44, (1988).

(30) Grinstein, F.F. and DeVore, C.R., On Global Instabilities in Countercurrent Jets, Physics of Fluids, Vol.14,
No.3 (2002), pp.1095-1100.

(31) Dimotakis, P.E. and Brown, G.L., The Mixing Layer at High Reynolds Number: Large-Structure Dynamics and Entrainment, J. Fluid Mech., Vol.78 (1976), pp.535-560.

(32) Laufer, J. and Monkewitz, P.A., On Turbulent Jet Flows: A New Perspective, AIAA Paper 80-0962, Hartford, (1980).

(33) Ho, C.M. and Nosseir, N.S., Dynamics of an Impinging Jet. Part 1. The Feedback Phenomenon, J. Fluid Mech., Vol.105 (1981), pp.119-142.

(34) Gutmark, E.J. and Ho, C.M., Preferred Modes and the Spreading Rates of Jets, Phys. Fluids, Vol.26, No.10 (1983), pp.2932-2938.

(35) Gutmark, E.J. and Grinstein, F.F., Flow Control with Noncircular Jets, Annu Rev. Fluid Mech., Vol.31 (1999), pp.239-272.

(36) Grinstein, F.F. and Guirguis, R.H., Effective Viscosity in the Simulation of Spatially Evolving Shear Flows with Monotonic FCT Models, J. Comp. Phys., Vol.101 (1992), pp.165-175.

(37) Hussain, F. and Husain, H.S., Elliptic Jets. Part I. Characteristics of Unexcited and Excited Jets, J. Fluid Mech., Vol.208 (1989), pp.275-320.

(38) Jiménez, J., Wray, A., Saffman, P. and Rogallo, R., The Structure of Intense Vorticity in Isotropic Turbulence, J. Fluid Mech., Vol.255 (1993), pp.65-90.

(39) Porter, D.H., Pouquet, A. and Woodward, P.R., Kolmogorov-Like Spectra in Decaying ThreeDimensional Supersonic Flows, Phys. Fluids, Vol.6, No.6 (1994), pp.2133-2142.

(40) Saddoughi, S.G., Local Isotropy in High Reynolds Number Turbulent Shear Flows, CTR Annual Research Briefs, Stanford, USA, (1992), pp.237-262.

(41) Grinstein, F.F. and Fureby, C., Recent Progress on MILES for High Reynolds-Number Flows, J. Fluids Engineering, Vol.124 (2003), pp.848-861.

(42) Drikakis, D., Advances in Turbulent Flow Computations Using High-Resolution Methods, Progress in Aerospace Sciences, Vol.39 (2003), pp.405-424.

(43) Grinstein, F.F. and Fureby, C., From Canonical to Complex Flows: Recent Progress on Monotonically Integrated LES, Computing in Science \& Engineering, Vol.6, March-April (2004), pp.36-49. 